\title{
12 Months
}

National Cancer Institute

\section{Source}

National Cancer Institute. 12 Months. NCI Thesaurus. Code C156842.

A period of time of twelve months. 